\title{
Spontaneous Closure of Macular Hole Following the Separation of Vitreomacular Traction
}

\author{
Ayse Gul Kocak Altintas ${ }^{1^{*}}$ \\ ${ }^{1}$ Assosiate Professor, Saglik Bilimleri University, Ulucanlar Eye Hospital Ankara, Turkey \\ "Corresponding Author: Ayse Gul Kocak Altintas, Assosiate Professor, Saglik Bilimleri University, Ulucanlar \\ Eye Hospital Ankara, Turkey; Email: aysegulkaltintas@ @otmail.com
}

Received Date: 11-03-2021; Accepted Date: 20-04-2021; Published Date: 27-04-2021

Copyright $^{\oplus} 2021$ by Altintas AGK. All rights reserved. This is an open access article distributed under the terms of the Creative Commons Attribution License, which permits unrestricted use, distribution, and reproduction in any medium, provided the original author and source are credited.

\begin{abstract}
A 65-year-old man had continuously increased VMT that progress to symptomatic IMH during the treatment of fellow eye for AMD was presented. Spontaneous closure of IMH following detachment of VMT and completely resolved macular edema associated with recovery of the foveal structure observed by OCT, after four years follow up period.
\end{abstract}

\section{Keywords}

Posterior Vitreous Detachment; Retinal Distortion; Retina; Idiopathic Macular Holes

\section{Introduction}

Normal aging of the eye is carried out by several progressive degenerative changes in the vitreous body. This process is characterized by the liquefaction of vitreous gel associated with the posterior vitreous cortex's separation from the retinal surface. Incomplete Posterior Vitreous Detachment (PVD) at the macula without traction is described as Vitreomacular Adhesion (VMA), while persistent strong adherence at the macula associated with traction is described as Vitreomacular Tractional (VMT) [1-3]. Tractional forces in VMT cause anatomical changes such as retinal distortion, intraretinal pseudocyst, foveal splits and may lead to the development of a full-thickness Macular Hole (MH) in most cases. Spontaneous regression of VMT and closure of MH was reported in a small number of cases [1-6]. 
In this case report, we report a case that a spontaneous separation of VMT combined with the closure of symptomatic stage 1 macular hole and recovery of the foveal morphological destruction after five years during treatment of aged related macular degeneration on his other eye.

\section{Case Report}

A 65-year-old man presented to the hospital with decreased central vision with a central scotoma in the Right Eye (RE) in January 2016. He was diagnosed with Age-Related Macular Degeneration (AMD) in his RE and incomplete posterior vitreous detachment in his Left Eye (LE), which was observed by OCT (Fig. 1) His best-corrected visual acuity was 0.2 in the RE and 1.0 in his LE measured by Snellen chard. He underwent a series of intravitreal AntiVascular Endothelial Growth Factor (Ani-VEGF) treatment for AMD in RE and regular follow-up for both eyes. During his treatment for his RE and regular control for LE, the asymptomatic VMA progress to VMT in two years (Fig.2 and 3). A moderate decrement in VA without metamorphopsia was occurred correlated with the presence of VMT in his LE. His VA was decreased by 0.8 , while increment of macular thickness combined with hard drusens were observed by OCT. His VA and OCT findings on his LE stayed stable until January 2020. But due to the COVID-19 pandemic, he discontinued to regular examination and treatment. In November 2020, he presented to the retina clinic with metamorphopsia, and decreased vision. On his examination, VA was reduced in finger counting from $5 \mathrm{~m}$ in his RE and 0.1 in LE eye. OCT evaluation revealed the macular scar on his RE and stage 1b Idiopathic Macular Holes (IMH) with persistent VMT, distortion of retinal tissue, and increment of the macular thickness in his LE (Fig. 4). Vitreoretinal surgery was recommended to release VMT and prevent a fullthickness macular hole for LE. But due to his personal problems during the COVID pandemic, the surgery was postponed. In January 2021, he presented to the retina clinic with increased vision and decreased visual distortion. His VA increased to 0.4 , and spontaneous separation of VMT, closure of MH combined with significant decreased in macular thickness observed by OCT (Fig. 5). During the follow- up examination, recovery of the foveal structure with reorganization of residual photoreceptor Ellipsoid Zone (EZ) disorganization was observed. 


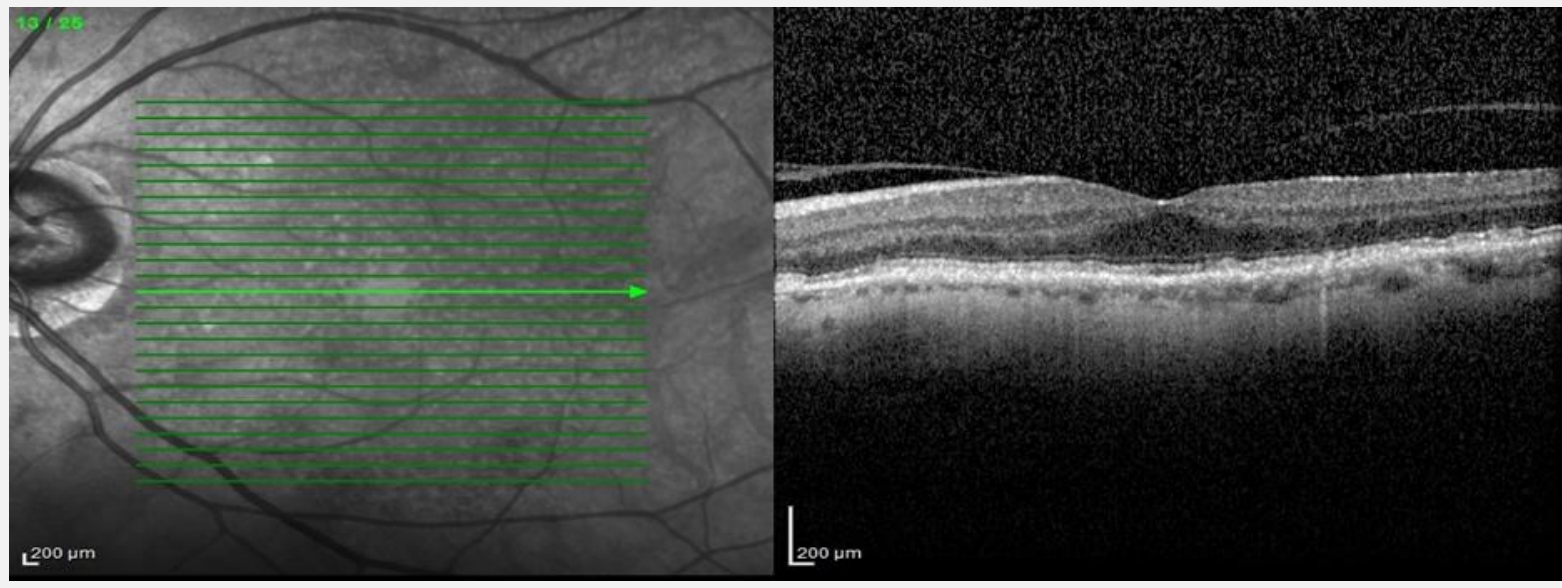

Figure 1: Incomplete Posterior Vitreous Detachment (PVD).

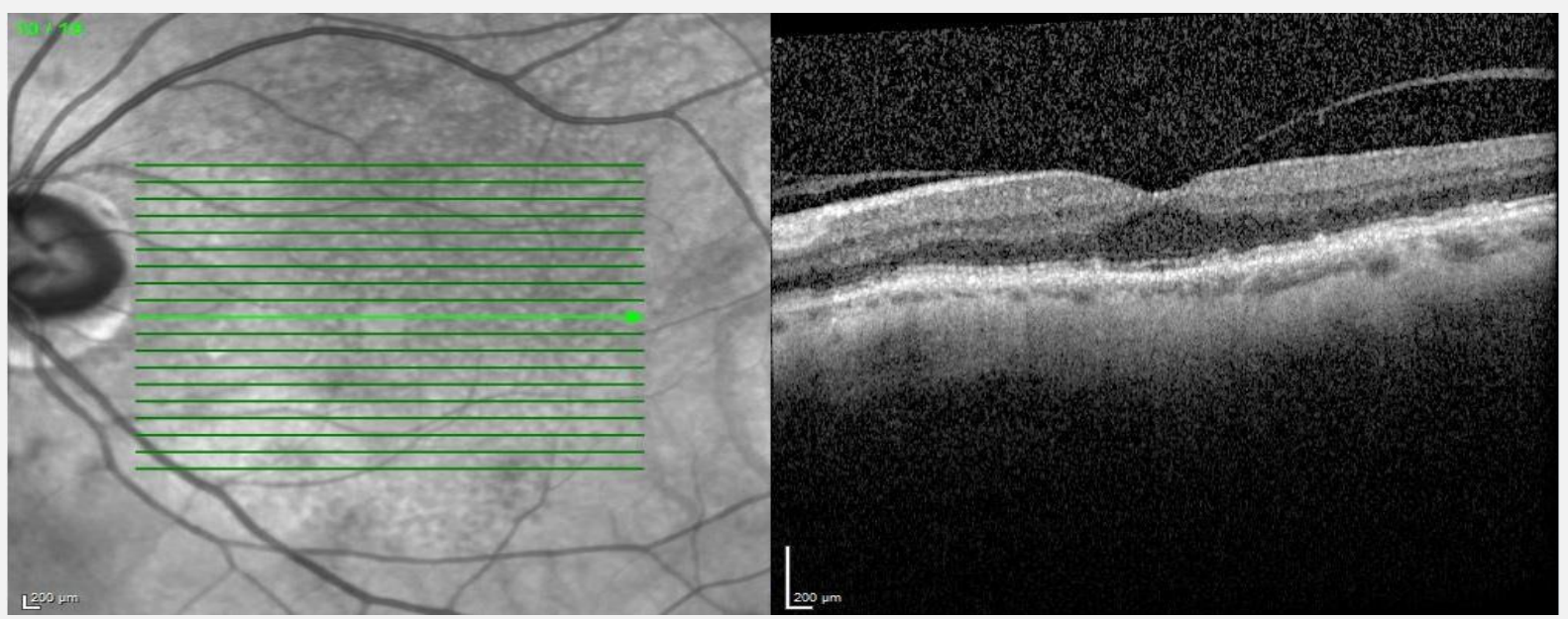

Figure 2: Incomplete PVD with minimal VMT.

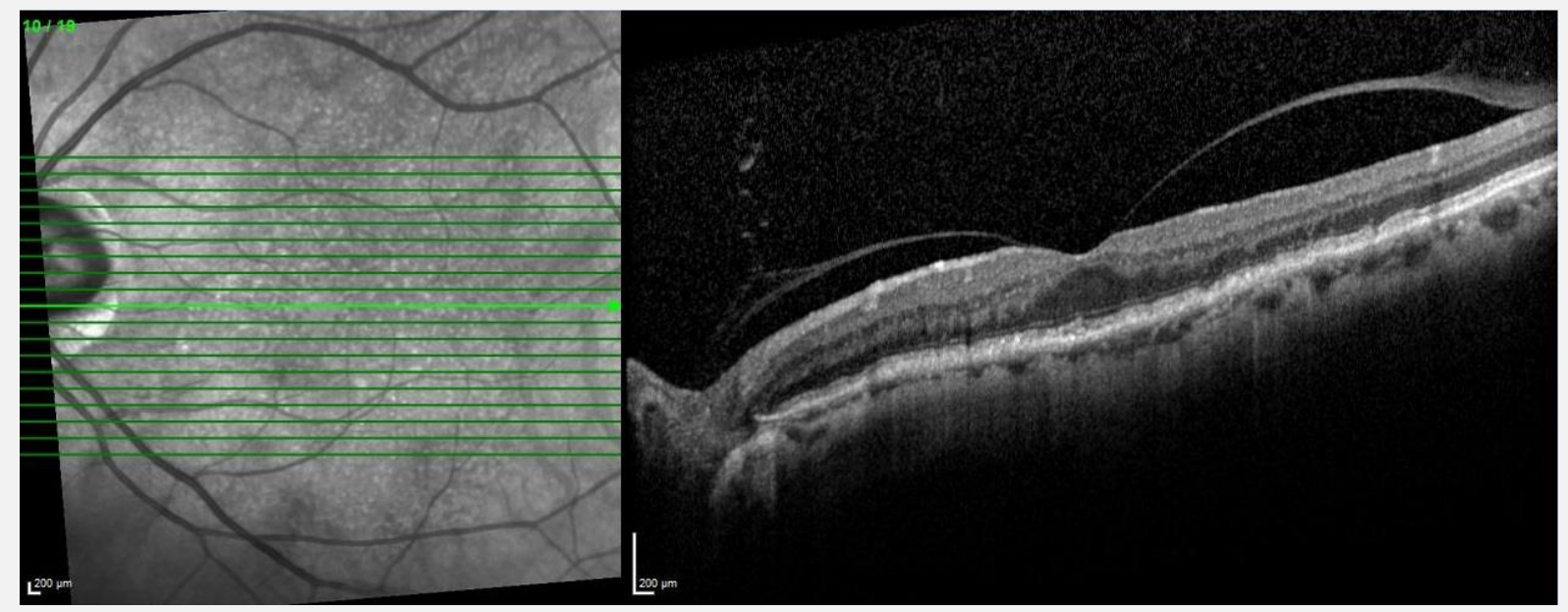

Figure 3: Persistance of Incomplete PVD with minimal VMT.

Altintas AGK | Volume 2; Issue 1 (2021) | JOAR-2(1)-012 | Case Report

Citation: Altintas AGK. Spontaneous Closure of Macular Hole Following the Separation of

Vitreomacular Traction. J Ophthalmol Adv Res. 2021;2(1):1-7.

DOI: http://dx.doi.org/10.46889/JOAR.2021.2107 


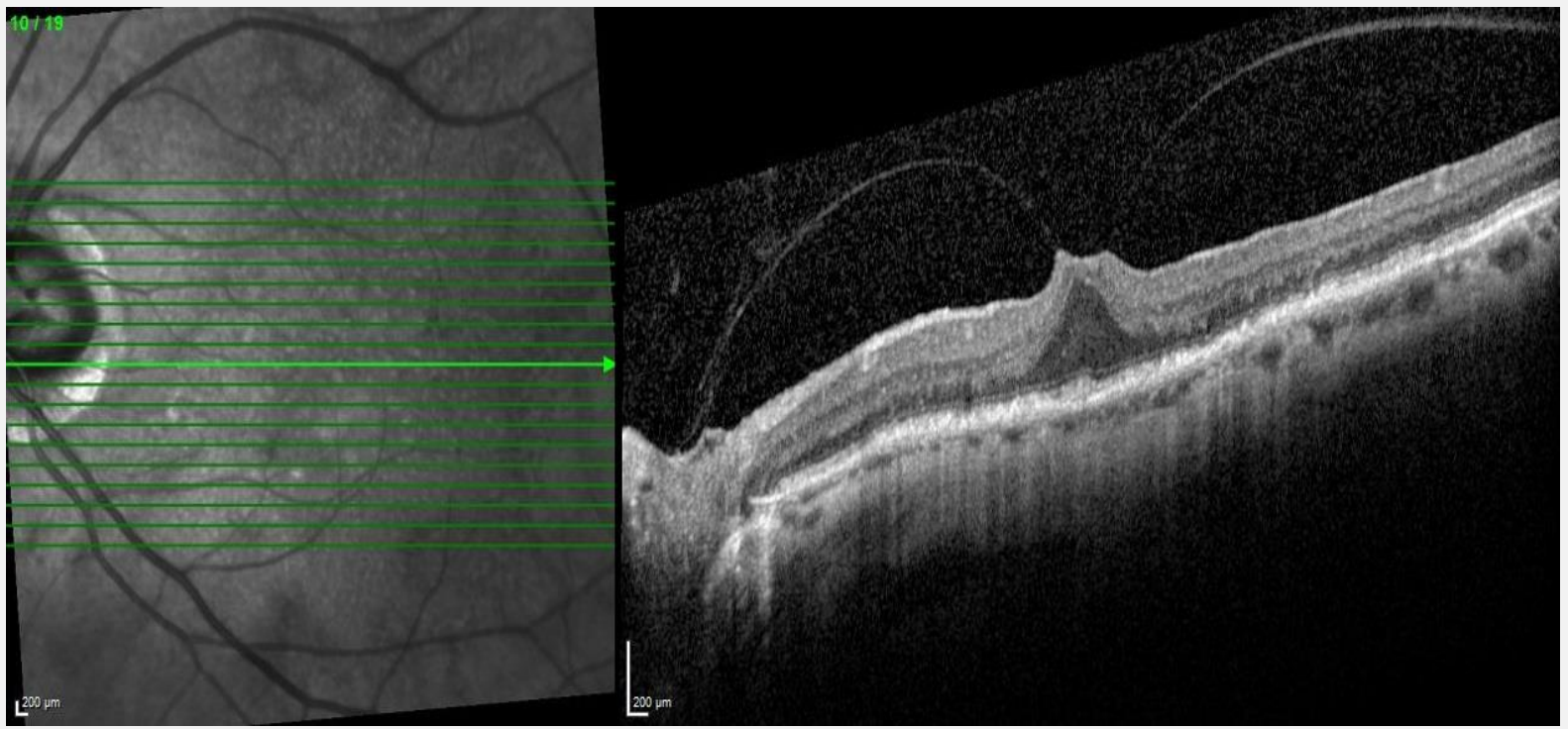

Figure 4: VMT with increment of the macular thickness.

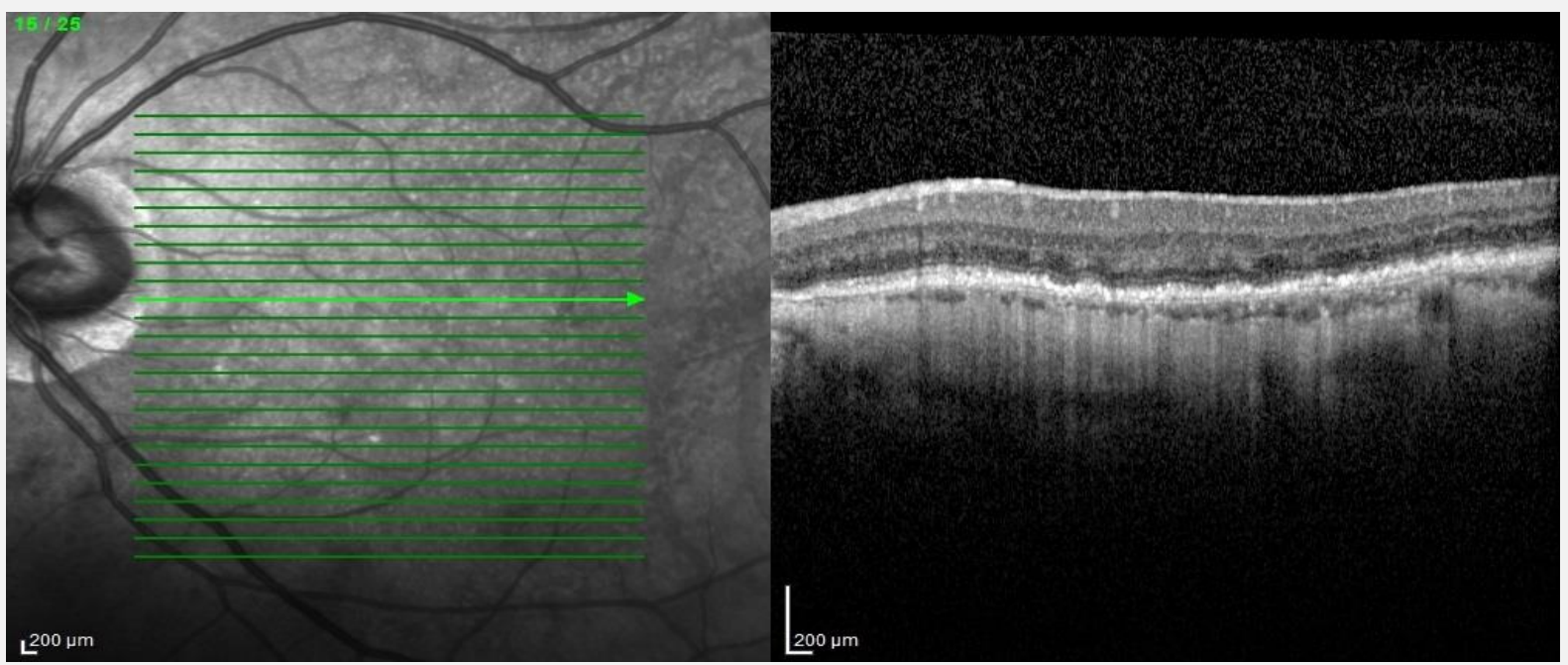

Figure 5: Spontaneous separation of VMT, combined with significant decreased in macular thickness.

\section{Discussion}

In this report, we demonstrate an asymptomatic case with continuously increased VMT progresses to symptomatic stage $1 \mathrm{IMH}$ in a four-year period during treatment of a fellow eye for AMD. We observed spontaneous closure of IMH with recovery of the foveal anatomy after detachment of VMT by OCT evaluation.

Normal age-related PVD generally results in innocuous separation of vitreous from the entire retinal surface. But, in incomplete PVD, VMT may exert tractional forces on the macula, which leads to distortion of the retina and complicated by $\mathrm{MH}$ [1,6-7]. It has been reported that the

Altintas AGK | Volume 2; Issue 1 (2021) | JOAR-2(1)-012 | Case Report

Citation: Altintas AGK. Spontaneous Closure of Macular Hole Following the Separation of

Vitreomacular Traction. J Ophthalmol Adv Res. 2021;2(1):1-7. 
estimated prevalence of IMH is $0.1-0.8 \%$ and prevalence of VMT in IMH is 22.5 per 100000 of the adult population [1,2,8-10].

Progression of VMT is hard to predict, therefore observation in an asymptomatic patient with stage 1 macular hole is essential as the standard of care. At least three months of follow-up is the first choice for many retinal surgeons before initiating treatment to avoid unnecessary intervention $[4,6,11,12]$. In the presented case, we observed the progression of VMT by OCT 4 years regularly as more than the recommended period. We prefer observation as long as slow progression without visual symptoms. Treatment is advised for late-stage 1 IMH that demonstrates progression to stage 2 IMH with persistent VMT, increased MT, presence of pseudocyst, and associated with clinical symptoms such as metamorphopsia and severe visual impairment $[1,4,6,11,12]$. When our case became symptomatic, and the traction was increased, we prefer to perform pars plana vitrectomy. But due to the COVID-19 pandemic and his personal problem, the surgery had to delay.

The incidences of spontaneous separation of VMT without intervention were reported 10-11\% $[1,8,9,13,14]$. In the Eye Disease Case-Control Study, Spontaneous regression or resolution of IMH was reported in $9 \%$ of patients after six years of follow-up. But the deterioration in VA of two Snellen lines or more was reported in $30 \%$ of patients with stage 1 over a period of at least one year in the same study [9].

Takahashi, et al., reported two eyes with foveal splits progressed to a full-thickness macular hole and 4 developed pseudocyst out of 7 eyes with incomplete VMT [15]. Niwa, et al., observed the change in the vitreo-foveal relationship in $47 \%$ and the development of an idiopathic full-thickness macular hole in $5.2 \%$ of eyes with in the follow-up period of 24 months [16].

Spontaneous closure of macular holes, mostly in advanced stages is uncommon; most of them reported as single cases or very small case series [6,13,14,17]. Ozgonul and Berlin reported a case spontaneous closure of stage $1 \mathrm{IMH}$ and completely resolved pseudocyst at month 3 [18]. Ohayon and Rubowitz reported a rare case with a spontaneous resolution of bilateral FTMH and posterior retinal layer re-organization [19].

The most accepted etiology of macular hole closure is completing incomplete PVD and separation of VMT that allows resolution of the cystic spaces and formation of normal anatomic foveal contour [13,20,21].

In this report, we presented a rare case with a spontaneous resolution of impending stage $2 \mathrm{MH}$ by the spontaneous release of long-standing VMT and re-organization of normal macular anatomy demonstrated by OCT. As far as we know, the presented case was one of the longest $\mathrm{IMH}$ that showed spontaneous resolution in the literature. 


\section{Conclusion}

In conclusion, because it is hard to predict the progression of VMT, regular evaluation of macula with OCT is essential for timely diagnosis for vitreoretinal interface pathology, detection of anatomical changes, and prevent unnecessary invasive procedures.

\section{References}

1. Steel DHW, Lotery AJ. Idiopathic vitreomacular traction and macular hole: a comprehensive review of pathophysiology, diagnosis, and treatment Eye (Lond). 2013;27(1):S1-21.

2. Menzler J, Neubauer AS, Haritoglou C, Jackson TL. Incidence and prevalence of vitreomacular traction with and without the macular hole in Germany. Clin Ophthalmol. 2019;13:177-88.

3. Kampik A. Pathology of epiretinal membrane, idiopathic macular hole and vitreomacular traction syndrome. Retina. 2012;32:S194-99.

4. Errera MH, Liyanage SE, Petrou P. A study of the natural history of vitreomacular traction syndrome by OCT. Ophthalmol. 2018;125:701-7.

5. Bottos J, Elizalde J, Rodrigues EB, Maia M. Current concepts in vitreomacular traction syndrome. Curr Opin Ophthalmol. 2012;23:195-201.

6. Dimopoulos S, Bartz-Schmidt KU, Gelisken F, Januschowski K, Ziemssen F. Rate and timing of spontaneous resolution in a vitreomacular traction group: should the role of watchful waiting be reevaluated as an alternative to ocriplasmin therapy? Br J Ophthalmol. 2015;99(3):350-3.

7. Majji $\mathrm{AB}$, Chhablani JK, Bagga B. Vitreo-retinal interface changes on optical coherence tomography in the fellow eyes of patients with macular hole. Int J Ophthalmol. 2013;6(4):526-30.

8. Kumagai K, Ogino N, Hangai M, Larson E. Percentage of fellow eyes that develop full-thickness macular hole in patients with unilateral macular hole. Arch Ophthalmol. 2012;130:393-4.

9. Chew EY, Sperduto RD, Hiller R, Nowroozi L, Seigel D, Yanuzzi LA, et al. Clinical course of macular holes. The eye disease case-control study. Arch Ophthalmol. 1999;117:242-6.

10. McCannel CA, Ensminger JL, Diehl NN, Hodge DN. Population based incidence of macular holes. Ophthalmol. 2009;116:1366-9.

11. Odrobina D, Michalewska Z, Michalewski J, Dziegielewski K, Nawrocki J. Long-term evaluation of vitreomacular traction disorder in spectral-domain optical coherence tomography. Retina. 2011;31:324-31.

12. Jackson TL, Nicod E, Simpson A, Angelis A, Grimaccia F, Kanavos P. Symptomatic vitreomacular adhesion. Retina. 2013;33:1503-11.

13. Weinand F, Jung A, Becker R, Pavlovic S. Spontaneous resolution of vitreomacular traction syndrome. Ophthalmologe. 2009;106:44-6.

14. Milani P, Seidenari P, Carmassi L, Bottoni F. Spontaneous resolution of a full-thickness idiopathic macular hole: fundus autofluorescence and OCT imaging. Graefes Arch Clin Exp Ophthalmol Albrecht Von Graefes Arch Klin Exp Ophthalmol. 2007;245(8):1229-31.

15. Takahashi A, Nagaoka T, Yoshida A. Stage 1-A macular hole: a prospective spectral-domain optical coherence tomography study. Retina. 2011;31(1):127-47.

16. Niwa H, Terasaki H, Ito Y, Miyake Y. Macular hole development in fellow eyes of patients with unilateral macular hole. Am J Ophthalmol. 2005;140(3):370-5.

17. Theodossiadis GP, Grigoropoulos VG, Theodoropoulou S, Datseris I, Theodossiadis PG. Spontaneous resolution of vitreomacular traction demonstrated by spectral-domain optical coherence tomography. American J Ophthalmol. 2014;157(4):842-51.

18. Ozgonul C, Besirli CG. Macular hole closure following spontaneous release of vitreomacular traction. BMJ Case Rep. 2017;2017:bcr2016218547.

Altintas AGK | Volume 2; Issue 1 (2021) | JOAR-2(1)-012 | Case Report

Citation: Altintas AGK. Spontaneous Closure of Macular Hole Following the Separation of Vitreomacular Traction. J Ophthalmol Adv Res. 2021;2(1):1-7. 
19. Ohayon A, Rubowitz A. Spontaneous closure of bilateral macular holes. Am J Ophthalmol Case Rep. 2019;15:100516.

20. García-Medina JJ, Del-Río-Vellosillo M, Rubio-Velázquez E, López-Bernal, Zafra-Pérez JJ. Focal vitreomacular traction: Resolution after ocular massage. Am J Ophthalmol Case Rep. 2019;14:61-3.

21. Dítě J, Štěpánková J, Burdová Č, Dotřelová D. The importance of evaluating the development of OCT findings during conservative treatment of vitreomacular traction complicated by macular hole formation. Ceska a Slovenska Oftalmologie: Casopis Ceske Oftalmologicke Spolecnosti a Slovenske Oftalmologicke Spolecnosti. 2019;74(6):240-4. 